\title{
Comparison of iso-effective and cost-effective high- dose-rate brachytherapy treatment schedules in cervical cancer - regional cancer center experience
}

\author{
Purnima Thakur, MD!, Ekta Dogra, MD², Prof. Manish Gupta, MD!, Rati Ram Negi, MD, Assoc. Prof. ${ }^{3}$, \\ Vikas Fotedar, MD, Assoc. Prof., Shalu Thakur, BScl, Chitranjan Sharma, BScl \\ 'Department of Radiotherapy, Indira Gandhi Medical College, Shimla, Himachal Pradesh, India, ²Department of Community Medicine, \\ PGIMER, Chandigarh, India, ${ }^{3}$ Department of Radiotherapy, Sri Lal Bahadur Shastri Government Medical College, Ner Chowk, Mandi, \\ Himachal Pradesh, India
}

\begin{abstract}
Purpose: The study is to evaluate the difference between outcomes of two high-dose-rate fractionation schedules in the treatment of intracavitary brachytherapy (ICBT) of cervical cancer.

Material and methods: A retrospective analysis of 163 cervical cancer patients was completed. All patients received external beam radiotherapy (EBRT) to whole pelvis with concurrent weekly chemotherapy, followed by ICBT with either 7 Gy per fraction in three fractions (arm A) or 9 Gy per fraction in two fractions (arm B). Median follow-up was 19 months. The outcomes were compared in terms of 2-year actuarial local control, disease-free survival, overall survival, and late toxicity in the two treatment arms.

Results: The 2-year actuarial local control rates in arm A and arm B were $88.5 \%$ and $91.5 \%$, respectively. The actuarial 2-year disease-free survival rates in arm A and arm B were 85.9\% and 82.6\%, respectively. The actuarial 2-year overall survival in arm A and arm B were $95.7 \%$ and $100 \%$, respectively $(p=0.06)$. There were $12.7 \%$ and $15.2 \%$ local failures in arm A and arm B, respectively. Distant metastases were seen in $8.5 \%$ and $7.6 \%$ in arm A and arm B, respectively. The 2-year actuarial risk of developing late rectal toxicity in arm A and arm B were $5.6 \%$ and $5.4 \%$, respectively. The 2-year actuarial risk of developing late bladder toxicity in arm A and arm B were $2.8 \%$ and 2.2\%, respectively.

Conclusions: ICBT treatment with 9 Gy in two fractions offers equivocal local control rates and survival rates in cancer cervix cases with many advantages of short overall treatment time, improved patient compliance, cost effectiveness, and reduced exposure to aesthetic agents. The toxicities observed were few, low grade, and easily manageable.

J Contemp Brachytherapy 2019; 11, 5: 428-435 DOI: https://doi.org/10.5114/jcb.2019.88329
\end{abstract}

Key words: brachytherapy, high-dose-rate schedules, cancer cervix.

\section{Purpose}

Cervical cancer is the fourth most common cancer in women and the eighth most common cancer overall worldwide [1,2]. It is the cause of third largest cancer mortality in India, accounting for nearly $10 \%$ of all cancer-related deaths in the country $[3,4]$. Rural women are at higher risk of developing cervical cancer as compared to their urban counterparts [5].

There are 96,322 new cases of cervical cancer in India every year. At our institute, carcinoma of uterine cervix accounts for approximately $58.7 \%$ of all gynecological malignancies, with about $80-90 \%$ of patients presenting in locally advanced stage with bulky central disease. Thus, radiation therapy remains the preferred treatment modality in majority of patients.
Optimal treatment requires a combined approach including intracavitary radiation for in situ tumor and external radiation for parametrial tissue and pelvic draining lymph nodes. High-dose-rate (HDR) brachytherapy is now the standard brachytherapy modality used almost worldwide. It allows integration of external beam radiation therapy (EBRT) with brachytherapy as a sandwich regimen, reducing overall treatment time and providing better tumor control.

Even though published data of more than four decades are available, there is no consensus regarding optimal dose fractionation schedules of HDR brachytherapy for cancer cervix treatment.

A large number of different fractionation schedules of HDR ICBT (intracavitary brachytherapy) have been used varying from institution to institution, but an opti- 
mum schedule has not been established. The American Brachytherapy Society (ABS) has recommended an individual fraction size of less than 7.5 Gy in four to eight fractions, depending on the dose per fraction. These guidelines come with a word of caution stating that: "these recommendations are intended as guidelines and the suggested fractionation schemes have not been thoroughly tested" [6]

HDR fraction size of more than 7.5 Gy has been used in several centers, supported by findings of few studies $[7,8,9]$. There is a compelling need to increase the fraction size of HDR ICBT and thus, reduce the treatment time, keeping in view the ever-increasing patient load in Indian set up and limited availability of infrastructure.

Considering the high burden of disease and prolonged waiting time for ICBT, 9 Gy per fraction in two fractions and 7 Gy per fraction in three fractions are the two schedules followed at our institute.

With the aim to evaluate the difference between outcomes of two HDR fractionation schedules, a retrospective analysis of 163 cancer cervix patients was done. The outcomes were compared in terms of 2-year actuarial local control, disease-free survival, overall survival, and late toxicity: arm A (control) - 7 Gy per fraction in three fractions and arm B (study) - 9 Gy per fraction in two fractions.

\section{Material and methods}

This was a mono-institutional retrospective study conducted at the Regional Cancer Center, located at the Northern Himalayan region of India.

ICBT facility is available only in our institution in the State. Therefore, considering the high burden of disease and prolonged waiting time for ICBT, 9 Gy per fraction in two fractions and 7 Gy per fraction in three fractions are the two schedules followed at our institute.

We analyzed 163 patients of cancer cervix stage IB to stage IIIB, who underwent treatment from February 2016 to August 2018, including patients in follow-up till $30^{\text {th }}$ January 2019. Institutional ethical committee clearance was obtained before commencement of the study. Age group of patients ranged from 35 years to 80 years.

All patients received EBRT delivered to whole pelvis by ${ }^{60} \mathrm{Co}$ by Theratron or Equinox by either two field or four field technique, depending on patient separation. Radiotherapy planning was completed on conventional simulator.

EBRT dose was either 45 Gy in 25 fractions or 50 Gy in 25 fractions in 5 weeks (1.8 Gy or 2 Gy per fraction, respectively, 5 fractions/week), with central shielding in last 3 fractions in patients receiving 50 Gy to spare bladder and rectum.

Additionally, all patients received concurrent weekly injection of cisplatin $40 \mathrm{mg} / \mathrm{m}^{2}$ as a radiosensitizer with EBRT. Chemotherapy was planned for 5 cycles, but due to chemotherapy-induced acute toxicities, only 9 patients received 3 to 4 cycles of chemotherapy.

After completion of EBRT, patients received HDR ICBT in two treatment schedules: 71 patients in arm A received 7 Gy per fraction in three fractions, with 1-week interval between fractions, and 92 patients in arm B re- ceived 9 Gy per fraction in two fractions, with 1-week interval between fractions. Orthogonal anteroposterior and lateral simulation $X$-rays were taken using dummy sources on conventional simulator. These films were used in Oncentra 3D treatment planning system for applicator reconstruction, defining point $\mathrm{A}$, prescribing point $\mathrm{A}$ dose, and calculating bladder and rectal doses as per ICRU 38 recommendations. HDR brachytherapy was delivered by HDR microSelectron, using ${ }^{192}$ Ir source by Fletcher Williamson Asia Pacific applicators.

The total biological effective dose (BED) delivered to point $\mathrm{A}$ in arm $\mathrm{A}$ was $\mathrm{BED}_{\mathrm{EBRT}}+\mathrm{BED}_{\mathrm{ICBT}}=[25 \times 2(1+$ $2 / 10)]+[3 \times 7(1+7 / 10)]=60+35.7=95.7$ Gy. Equivalent dose in $2 \mathrm{~Gy}$ fraction $\left(\mathrm{EQD}_{2}\right)$ to point $\mathrm{A}$ in arm $\mathrm{A}$ was $\mathrm{BED} /(1+2 / 10)=79.75 \mathrm{~Gy}$ (or $74 \mathrm{~Gy}$ with $1.8 \mathrm{~Gy}$ per fraction of EBRT).

Similarly, the total BED delivered to point A in arm $\mathrm{B}$ was $\mathrm{BED}_{\mathrm{EBRT}}+\mathrm{BED}_{\mathrm{ICBT}}=[25 \times 2(1+2 / 10)]+[2 \times 9$ $(1+9 / 10)]=60+34.2=94.2 \mathrm{~Gy} \cdot \mathrm{EQD}_{2}$ to point $\mathrm{A}$ in arm B was BED $/(1+2 / 10)=78.5$ Gy (or 72.75 Gy with $1.8 \mathrm{~Gy}$ per fraction of EBRT).

\section{Results}

The basic characteristics of the patients in the two treatment arms are depicted in Table 1.

The median age of patients in arm A was 55 years and that of arm B was 52.5 years. In both arms, the majority of patients were diagnosed as stage IIB, with $66.2 \%$ in arm $\mathrm{A}$ and $62.0 \%$ in arm B, respectively. Pre-treatment hemoglobin level was $>10 \mathrm{gm} / \mathrm{dl}$ in $87.3 \%$ and $76.1 \%$ cases in arm A and arm B, respectively (Table 1).

Median follow-up in arm A was 22 months (range, 6-33 months), and median follow-up in arm B was 19 months (range, 8.9-34 months). Five patients in arm A and two in arm B were lost to follow-up. Three patients died in arm A after treatment completion and no death occurred in arm B. The cause of deaths in arm A can be attributed to distant spread of the disease.

The 2-year actuarial local control rates in arm A and arm B were $91.5 \%$ and $89.1 \%$, respectively (Figure 1). Even though the local control rates were higher in arm A as compared to arm B, the difference between the two arms was not statistically significant $(p=0.3)$.

\section{Patterns of failure}

There were $12.7 \%$ (9 out of 71 ) and $15.2 \%$ (14 out of 92) local failures in arm A and arm B, respectively. Distant metastases were seen in $8.5 \%$ (6 out of 71 ) and $7.6 \%$ (7 out of 92) in arm A and arm B, respectively, mostly occurring in para-aortic lymph nodes and supraclavicular lymph nodes. Overall failure was seen in $38.1 \%$ and $43.5 \%$ of patients in arm A and arm B, respectively (Table 2).

\section{Disease-free survival}

The actuarial 2-year disease-free survival (DFS) rates in arm A and arm B were $88.7 \%$ and $84.8 \%$, respectively (Figure 2). Although, the disease-free survival was higher in arm A, there was no statistically significant difference $(p=0.5)$ when compared to that of arm B. 
Table 1. Patient characteristics in both the study arms

\begin{tabular}{|c|c|c|c|}
\hline Patients characteristics & $\begin{array}{c}\text { Arm A (7 Gy/session) } \\
n \text { patients (\%) }\end{array}$ & $\begin{array}{c}\text { Arm B (9 Gy/session) } \\
n \text { patients (\%) }\end{array}$ & $P$ value \\
\hline \multicolumn{3}{|l|}{ Age group (years) } & \multirow[t]{3}{*}{$0.23^{*}$} \\
\hline$<50$ & $27(38)$ & $42(45.7)$ & \\
\hline$>50$ & $44(62)$ & $50(54.3)$ & \\
\hline Mean age (years) & 54.5 & 53.6 & \\
\hline \multicolumn{4}{|l|}{ FIGO stage } \\
\hline$\overline{\mathrm{IB}}$ & $3(4.2)$ & $3(3.3)$ & \multirow[t]{6}{*}{$0.81^{5}$} \\
\hline$\| A$ & $2(2.8)$ & $2(2.2)$ & \\
\hline $\mathrm{IIB}$ & $47(66.2)$ & $57(62.0)$ & \\
\hline IIIA & $4(5.6)$ & $3(3.3)$ & \\
\hline $\mathrm{IIIB}$ & $15(21.1)$ & $26(28.3)$ & \\
\hline IVA & $0(0)$ & $1(1.1)$ & \\
\hline \multicolumn{4}{|l|}{ Histology } \\
\hline Squamous & $68(95.8)$ & $85(92.4)$ & \multirow[t]{2}{*}{$0.37^{\$}$} \\
\hline Adeno & $3(4.2)$ & $7(7.6)$ & \\
\hline \multicolumn{4}{|l|}{ Differentiation } \\
\hline Well & $17(23.9)$ & $15(16.3)$ & \multirow[t]{4}{*}{$0.64^{5}$} \\
\hline Moderately & $42(59.2)$ & $62(67.4)$ & \\
\hline Poorly & $10(14.1)$ & $12(13.0)$ & \\
\hline Unspecified & $2(2.8)$ & $3(3.3)$ & \\
\hline \multicolumn{4}{|l|}{ Fields } \\
\hline Two & $49(69)$ & $60(65.2)$ & \multirow[t]{2}{*}{$0.61^{*}$} \\
\hline Four & $22(31)$ & $32(34.8)$ & \\
\hline \multicolumn{4}{|c|}{ Pre-treatment Hemoglobin levels (gm/dl) } \\
\hline$<10$ & $9(12.7)$ & $22(23.9)$ & \multirow[t]{2}{*}{$0.07^{\star}$} \\
\hline$>10$ & $62(87.3)$ & $70(76.1)$ & \\
\hline
\end{tabular}

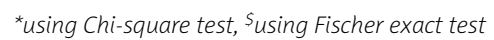



Fig. 1. Actuarial local control in 2 years, $p=0.3$

\section{Local response}

Complete response was achieved on first follow-up at six weeks in $73.2 \%$ and $67.4 \%$ in arm A and arm B, respectively $(p=0.53)$, as presented in Table 3 . In $\operatorname{arm} B$, two patients reported progressive disease, with one presenting vesicovaginal fistula (VVF) on assessing local response (LR) at first follow-up. The margins of VVF were everted and tested positive for malignancy. On subsequent follow-up, this patient developed distant metastasis in the lumbar spine, pelvic nodes, and lungs.

Acute gastrointestinal toxicities were reported in $14.1 \%$ and $18.5 \%$ in arm $\mathrm{A}$ and arm $\mathrm{B}$, respectively $(p=0.66)$. At the same time in arm A, acute hematological toxicities were reported amongst $16.9 \%$ and in $25 \%$ of cases in arm B $(p=0.35)$. The acute toxicities observed were mostly grade 1 and few of grade 2 . These were easily manageable, and patients tolerated the treatment well. None of the patients experienced higher grade acute toxicities. 
Table 2. Patterns of failure in the two brachytherapy treatment arms

\begin{tabular}{|c|c|c|c|}
\hline Patterns of failure & $\begin{array}{c}\text { Arm A (7 Gy/session) } \\
n \text { patients (\%) }\end{array}$ & $\begin{array}{c}\text { Arm B (9 Gy/session) } \\
n \text { patients (\%) }\end{array}$ & $P$ value \\
\hline Local failure & $9(12.7)$ & $14(15.2)$ & $0.9^{*}$ \\
\hline Distant failure & $6(8.5)$ & $7(7.6)$ & $0.8^{*}$ \\
\hline Local along with distant failure & $12(16.9)$ & $19(20.7)$ & $0.5^{\star}$ \\
\hline Overall failure & $27(38.1)$ & $40(43.5)$ & $0.06^{*}$ \\
\hline
\end{tabular}

*using Chi-square test

\section{Overall survival}

The actuarial 2-year overall survival (OS) rates in arm A and arm B were $95.7 \%$ and $100 \%$, respectively (Figure 3 ), although the difference did not reach any statistical significance $(p=0.06)$.

\section{Late toxicities}

Late toxicities were graded according to RTOG/ EORTC criteria (Table 4). Rectal toxicities were seen in 4 patients in arm A and in 5 patients in arm B. Although, there was an increased overall late toxicity in arm $B$ when compared to arm A, the difference was not statistically significant $(p=0.21)$. Grade III or more severe late toxicities were not seen in patients in both treatment groups. The dose was also calculated at ICRU rectal and bladder points. The median total rectal $\mathrm{EQD}_{2}$ in arm A from combined EBRT and brachytherapy sessions was 57.5 Gy (range, 49.5-67.8 Gy), and $57 \mathrm{~Gy}$ (range, 50.9-67.8 Gy) in arm B.

Grade 1 bladder toxicities were observed in 2 patients in both the arms. None of the patients experienced higher grade bladder toxicities in either treatment group.

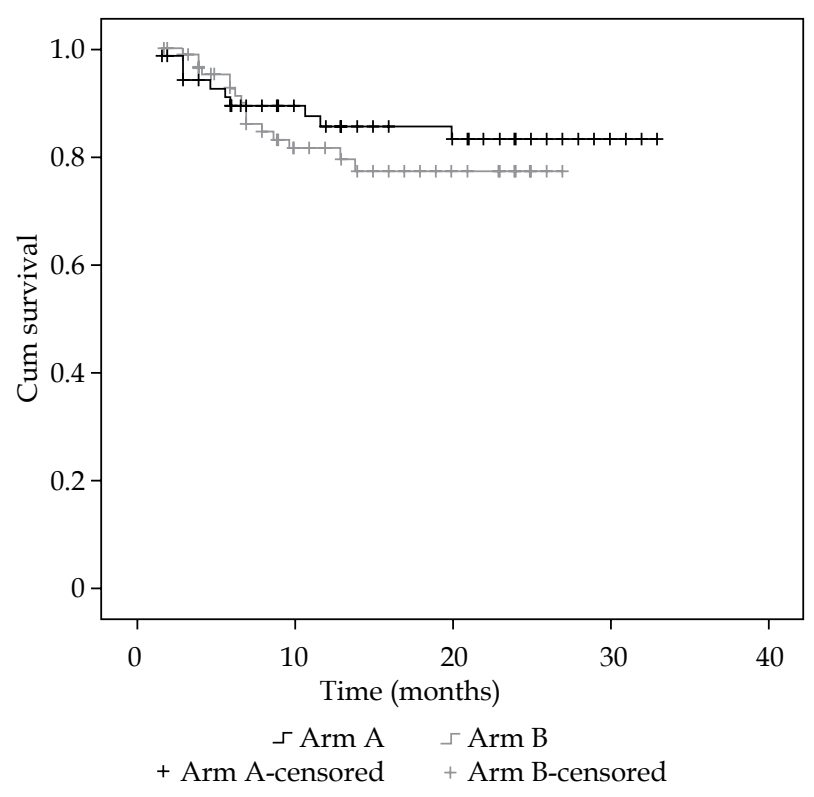

Fig. 2. Actuarial 2-year disease-free survival, Kaplan-Meier curve, $p=0.5$

Table 3. Local response at first follow-up and acute toxicities Local response at first follow-up

\begin{tabular}{|c|c|c|c|}
\hline Patterns of failure & $\begin{array}{c}\text { Arm A (7 Gy/session) } \\
n \text { patients (\%) }\end{array}$ & $\begin{array}{c}\text { Arm B (9 Gy/session) } \\
n \text { patients (\%) }\end{array}$ & $P$ value \\
\hline Complete response & $52(73.2)$ & $62(67.4)$ & $0.53^{*}$ \\
\hline Partial response & $18(25.4)$ & $27(29.3)$ & \\
\hline Progressive disease & $1(1.4)$ & $2(2.2)$ & \\
\hline Vesico-vaginal fistula & $0(0)$ & $1(1.1)$ & \\
\hline \multicolumn{4}{|l|}{ GI toxicities } \\
\hline Grade 1 & $5(7.0)$ & $7(7.6)$ & $0.66^{*}$ \\
\hline Grade 2 & $5(7.0)$ & $10(10.9)$ & \\
\hline \multicolumn{4}{|c|}{ Acute hematological toxicity } \\
\hline Grade 1 & $9(12.7)$ & $13(14.1)$ & $0.35^{\$}$ \\
\hline Grade 2 & $3(4.2)$ & $9(9.8)$ & \\
\hline Grade 3 & $0(0)$ & $1(1.1)$ & \\
\hline \multicolumn{4}{|l|}{ GU toxicity } \\
\hline Grade 1 & $2(2.8)$ & $6(6.5)$ & $0.9^{*}$ \\
\hline
\end{tabular}

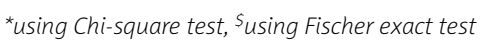






Fig. 3. Actuarial 2-year overall survival, $p=0.06$

The median total bladder $\mathrm{EQD}_{2}$ in arm A from combined EBRT and brachytherapy sessions was 55.9 Gy (range, 47.4-68 Gy), and 56.75 Gy (range, 49.4-73 Gy) in arm B.

No correlation was noted between the toxicity rate and the dose received at ICRU bladder and rectal points in both the arms.

When comparing late toxicities in terms of EBRT dose/fractionation schedules, six patients reported rectal toxicities in $50 \mathrm{~Gy} / 25$ fractions group and three patients in 45 Gy/25 fractions group, but $p$ value was not significant. Three patients experienced late bladder toxicity in $50 \mathrm{~Gy} / 25$ fractions group and one patient in the other EBRT schedule group. However, the difference was not statistically significant (Table 5).

The 2-year actuarial risk of developing any late rectal toxicity in arm A and arm B were $5.6 \%$ and $5.4 \%$, respectively. The 2-year actuarial risk of developing late bladder toxicity in arm A and arm B were $2.8 \%$ and $2.2 \%$, respectively.

The actuarial 2-year disease-free survival was also compared in the two treatment groups in the early and locally advanced disease. Stages IA, IIA, and IIB were included in early stage, and stages III and IVA was included in locally advanced disease for evaluation of results. In early stage, two graphs could not be drawn since in both groups, only one event of each occurred, i.e., both $80 \%$. In locally advanced stage, 2-year disease-free survival was better in arm A $(89.4 \%)$ than arm B $(85.1 \%)$, but the difference was not statistically significant $(p=0.4)$ (Figure 4 ).

\section{Discussion}

With availability of documented evidences, the treatment of cervical cancer is evolving. Despite the trends of declining utilization, brachytherapy is an integral component in the treatment of cancer cervix. ICBT has the advantage of delivering high dose to the tumor and restricting the dose to normal nearby structures, such as bladder and rectum. Therefore, for adequate tumor control, ICBT is irreplaceable.

Henschke et al. and O'Connell et al. introduced HDR remote afterloading brachytherapy in early 1960 [10,11]. Nowadays, HDR brachytherapy has replaced LDR

Table 4. Late toxicities in the two arms

\begin{tabular}{|c|c|c|c|c|c|}
\hline Patterns of failure & $\begin{array}{c}\text { Arm A (7 Gy/session) } \\
n \text { patients (\%) }\end{array}$ & $\begin{array}{l}\text { Median time to } \\
\text { toxicity (months) }\end{array}$ & $\begin{array}{c}\text { Arm B (9 Gy/session) } \\
n \text { patients }(\%)\end{array}$ & $\begin{array}{l}\text { Median time to } \\
\text { toxicity (months) }\end{array}$ & $P$ value \\
\hline \multicolumn{6}{|l|}{ Rectal toxicity } \\
\hline Grade 1 & $1(1.4)$ & \multirow[t]{3}{*}{10.25} & $4(4.3)$ & \multirow[t]{3}{*}{11.7} & \multirow[t]{3}{*}{$0.21^{5}$} \\
\hline Grade 2 & $3(4.2)$ & & $1(1.1)$ & & \\
\hline Grade 3 & $0(0)$ & & $0(0)$ & & \\
\hline \multicolumn{6}{|l|}{ Bladder toxicity } \\
\hline Grade 1 & $2(2.8)$ & \multirow[t]{2}{*}{19.5} & $2(2.2)$ & \multirow[t]{2}{*}{18.2} & \\
\hline Grade 2 & $0(0)$ & & $0(0)$ & & \\
\hline
\end{tabular}

*using Chi-square test, Susing Fischer exact test

Table 5. Late toxicities in the two treatment arms as per EBRT dose

\begin{tabular}{lccc} 
Patterns of failure & $\begin{array}{c}\text { EBRT dose } 45 / 25 \\
n \text { patients }(\%)\end{array}$ & $\begin{array}{c}\text { EBRT dose } 50 / 25 \\
n \text { patients (\%) }\end{array}$ & $P$ value \\
\hline Rectal toxicity & & & $0.94^{5}$ \\
\hline Arm A & $1(2.8)$ & $3(8.3)$ & \\
\hline Arm B & $2(11.6)$ & $3(4.0)$ & $0.25^{5}$ \\
\hline Bladder toxicity & $1(2.8)$ & $1(2.8)$ &
\end{tabular}


brachytherapy considering the advantages of delivering high dose in less time, better geometric placement, no hospital admission required, reduced immobilization time, lesser number of medical personnel needed, and decreased radiation to health care workers. Viani et al. in a meta-analysis of 5 RCT (2,065 patients) comparing HDR to LDR in cervical cancer cases showed no significant differences between HDR and LDR for OS, LR, and late complications for clinical stage I, II, and III [12]. Lertsanguansinchai et al. in a phase III randomized trial with 237 patients showed comparable outcomes between LDR and HDR intracavitary brachytherapy in cancer cervix. The 3-year pelvic control rates were $89.1 \%$ and $86.4 \%$ in LDR and HDR groups, respectively [13].

The efficacy of HDR brachytherapy can be improved by using 3D MRI-guided brachytherapy (IGBT). The use of 3D brachytherapy technique presented a trend towards an increased local control and improved overall survival with reduced toxicity, compared to the conventional 2D brachytherapy technique [14].

The aim of our present study was to compare two treatment schedules of HDR ICBT in cancer cervix in terms of late toxicities, local control, disease-free survival, and overall survival rates. In the past, many studies have compared higher dose per fraction with ABS recommended schedule, and the presented results are rewarding $[7,8,9,15]$.

According to a survey conducted by Bandyopadhyay et al. amongst young radiation oncologist of India regarding treatment of locally advanced carcinoma cervix, the most common brachytherapy dose patterns practiced for all stages were $7 \mathrm{~Gy}$ in 3 fractions and $9 \mathrm{~Gy}$ in 2 fractions. They concluded, that although fractionation patterns may vary, the overall mean dose administered for cervical cancer is similar across the country, which is slightly lower than the recommended doses per stage by various international guidelines [16].

Sharma et al. provided the results of 42 locally advanced cervical cancers patients treated with two weekly sessions of HDR-ICBT, with 10 Gy each delivered 1 week after pelvic EBRT. The 3-year overall survival rates for all stages were $47 \%$, and the 3-year recurrence-free survival for stage IIB, IIIB, and IVA were $67 \%, 34 \%$, and $20 \%$, respectively [17]. Clinical results have shown that weekly HDR-ICBT schedule $(2 \times 10 \mathrm{~Gy})$ is associated with low toxicity, decent local control, and survival rates, thereby proving its clinical feasibility.

Patel et al. reported 5-year results of 121 patients of stage I to III cancer cervix treated with HDR brachytherapy with 9 Gy in two fractions, 1 week apart, interdigitated with EBRT. The 5-year actuarial local control and disease-free survival rates were $74.5 \%$ and $62.0 \%$, respectively. None of the patients developed grade 3 rectal toxicity. Grade 3 bladder toxicities were observed in 2 patients. The actuarial risk of grade 3 or worse late toxicity was $3.31 \%$. The authors concluded that HDR brachytherapy in cancer cervix at 9 Gy per fraction is both safe and effective, with good local control and acceptable normal tissue toxicity [7].

In another prospective randomized trial by the same author, the patients in HDR arm who received 2 frac-



Fig. 4. Actuarial 2-year disease-free survival at locally advanced stage in two arms, Kaplan-Meier curve, $p=0.4$

tions of 9 Gy per fraction had local pelvic control rates and 5-year DFS rates of $73.8 \%$ and $53.6 \%$, respectively. Rectal complications were noted in $6.9 \%$ of patients and incidence of more severe complications of grades 3 to 5 was at the level of $0.5 \%$. The bladder morbidity was $3.5 \%$ [18].

The ABS recommended schedules have not been thoroughly tested clinically. Due to paucity of clinical experience, bio effect dose models (mainly LQ model) has been used to convert HDR to LDR equivalent doses. However, clinical experience is far more superior to theoretical calculations for constituting treatment guidelines and can be implemented confidently. Thus, clinical studies are needed and HDR dose fractionation schedules are required to be tested, as this provides confidence in treating patients.

Orton et al. in an analysis of over 1,700 cervical cancer cases showed that morbidity rates were significantly lower in fraction size $<7$ Gy as compared to fraction size $>7 \mathrm{~Gy}$, but the cure rates in both groups were equivocal [19]. This suggests that normal tissue complication rates may be decreased by adequately using midline blocking techniques as well as proper vaginal packing and rectal retraction techniques.

Most of the data of HDR brachytherapy comes from developed countries, although cervical cancer is widely prevalent in developing countries. Cervical cancer is on the top of list of cancers in Indian females. Therefore, there is an urgent need to develop treatment guidelines suitable for our set up.

Patel et al. documented clinical experience of comparing their institutional protocol of 9 Gy per fraction in 2 fractions, one week apart, with a radiobiological equivalent dose of $6.8 \mathrm{~Gy}$ per fraction in total three fractions at weekly intervals. The 3-year actuarial LC rate for $9 \mathrm{~Gy}$ and 6.8 Gy arms $(81.35 \%$ vs. $65.18 \%$, respectively) and 
DFS rates (64.97\% vs. $49.47 \%$, respectively) were significantly better in the 9 Gy arm [9].

In a similar study on Indian patients by Saptrishi Ghosh, 7 Gy in 3 fractions schedule was compared to 9 Gy in 2 fractions. The author concluded that HDR ICBT with 9 Gy per fraction in two fractions is as effective as that of 7 Gy per fraction in three fractions, in terms of local control, disease-free survival, and overall survival. Although the late toxicities were higher with the 9 Gy per fraction HDR ICBT schedule when compared to that of 7 Gy per fraction, most of them were of low grade and thereby easily manageable [8].

The results of a randomized controlled trial comparing similar ICBT schedules are awaited [20].

There appears to be a possibility of reducing treatment time in cancer cervix. It is a well-known fact that reducing the treatment time in cervical cancer has a radiobiological significance, which converts into better tumor control. Fyles showed a loss of local control, approximating $1 \%$ per day for treatment prolongation of 30 days, especially in locally advanced stages (III-IV) [21]. Lanciano et al. reported a highly significant decrease in pelvic tumor control and survival with prolongation of treatment time. They described a 4-year actuarial infield recurrence rise from $6 \%$ to $20 \%$, when total treatment time increased from 6 weeks or less to 10 weeks $(p=0.0001)$ [22].

Treatment time in cancer cervix patients can be reduced by either interdigitating ICBT with EBRT or starting ICBT immediately after completion of EBRT. However, the majority of our patients present in locally advanced stage. In our study, more than $60 \%$ patients presented in stage IIB, and more than $28 \%$ patients presented in stage IIIB. Thus, interdigitation of ICBT with EBRT is insufficient due to anatomic distortion and bulky disease. Also, EBRT results in mucosal and skin toxicity, which becomes pronounced at the end of treatment and thus, requires a small interval for healing before ICBT could be started. In view of the above hurdles in early initiation of ICBT, the treatment time can be reduced by increasing brachytherapy fraction size. Thus, 9 Gy in 2 fractions remains an attractive option in a country like ours, where patients present in locally advanced stage, the burden of disease is high, and resources are limited. Lesser number of fractions will also reduce the cost of treatment.

Also, in a country like India, where infrastructure is limited and patient burden is ever-increasing, lesser number of HDR fractions will reduce brachytherapy waiting time and workload in an institute. Our institute caters the population of the whole state; therefore, there is a long waiting time for ICBT. Lesser number of HDR fractions will reduce this waiting time and further decrease the overall treatment time.

Few large fractions offer an additional advantage of improved patient compliance. It has been noticed in our institute that patients who are planned for 3 fractions of ICBT, usually default for third fraction. Patients come for treatment from various remote places, traveling in hilly areas every week for three or more weeks, which makes it difficult for patient to comply to treatment.
Khor et al., in a retrospective analysis, presented long-term results of 106 cervical cancer cases treated with HDR brachytherapy in a single center in Singapore. They summarized that the use of fewer fractions of HDR brachytherapy (compared with the ABS recommendations) with whole-pelvis XRT, without compromising tumor control, has positive economic implications for less developed countries having high cervical cancer prevalence and limited RT resources [23].

In our study, only 2 patients developed grade 1 bladder toxicity in 9 Gy arm and no patient developed higher grade. Also, grade 2 rectal toxicity was seen in 1 patient and grade 1 in 4 patients. This low rate of toxicities can be attributed to vaginal packing and rectal retractors (with ring applicators) judiciously used to displace the normal organs at risk as far as possible. The geometrical symmetry of applicators and vaginal and rectal packing is comfortably and effectively maintained, as HDR is a quick procedure. This advantage compensates for the radiobiologic loss of therapeutic ratio, when few large fractions are used.

However, two-year follow-up is sufficient to compare late toxicities, mainly rectal and bladder, but longer follow-up may lead to better evaluation of local control rates and overall disease-free survival rates. Thus, longer follow-up and more patient data may be needed to conclusively comment on the survival rates.

\section{Conclusions}

ICBT treatment with 9 Gy in two fractions offers equivocal local control rates and survival rates in cancer cervix cases, with many advantages including short overall treatment time, improved patient compliance, cost effective as less hospital admissions are required, and reduced exposure to anesthetic agents. Moreover, the toxicities observed are few, low grade, and easily manageable.

\section{Disclosure}

The authors report no conflict of interest.

\section{References}

1. Cervical cancer [Internet]. World Health Organization 2019 [cited 1 May 2019]. Available from: https://www.who.int/ cancer/prevention/diagnosis-screening/cervical-cancer/ en/

2. Cervical cancer statistics [Internet]. World Cancer Research Fund 2019 [cited 1 May 2019]. Available from: https:/ /www. wcrf.org/dietandcancer/cancer-trends/cervical-cancer-statistics

3. WHO. The global burden of disease: 2004 update [Internet]. Who.int. 2019 [cited 3 May 2019]. Available from: https:// www.who.int/healthinfo/global_burden_disease/2004_report_update/en/

4. [Internet]. Available from: http://screening.iarc.fr/doc/ WHO_India_CCSP_guidelines_2005.pdf

5. Kaarthigeyan K. Cervical cancer in India and HPV vaccination. Indian J Med Paediatr Oncol 2012; 33: 7-12.

6. Nag S, Erickson B, Thomadsen B et al. The American Brachytherapy society recommendations for high-dose-rate brachytherapy for carcinoma of the cervix. Int J Radiat Oncol Biol Phys 2000; 48: 201-211. 
7. Patel FD, Rai B, Mallick I et al. High-dose-rate brachytherapy in uterine cervical carcinoma. Int J Radiat Oncol Biol Phys 2005; 62: 125-130.

8. Ghosh S, Rao P. High-dose-rate orthogonal intracavitary brachytherapy with $9 \mathrm{~Gy} /$ fraction in locally advanced cervical cancer: is it feasible? J Obstet Gynaecol India 2016; 66 (Suppl 1): 452-458.

9. Patel FD, Kumar P, Karunanidhi G et al. Optimization of highdose-rate intracavitary brachytherapy schedule in the treatment of carcinoma of the cervix. Brachytherapy 2011; 2: 147-153.

10. Henschke U, Hilaris B, Mahan G. Remote afterloading with intracavitary applicators. Radiology 1964; 83: 344-345.

11. O'Connell D, Howard N, Joslin C et al. A new remotely controlled unit for the treatment of uterine carcinoma. Lancet 1965; 286: 570-571.

12. Viani GA, Manta G, Stefano E et al. Brachytherapy for cervix cancer: low-dose rate or high-dose rate brachytherapy a meta-analysis of clinical trials. J Exp Clin Cancer Res 2009; 28: 47 .

13. Lertsanguansinchai P, Lertbutsayanukul C, Shotelersuk K et al. Phase III randomized trial comparing LDR and HDR brachytherapy in treatment of cervical carcinoma. Int J Radiat Oncol Biol Phys 2004; 59: 1424-1431.

14. Derks K, Steenhuijsen JLG, Van den Berg HA et al. Impact of brachytherapy technique (2D versus $3 \mathrm{D}$ ) on outcome following radiotherapy of cervical cancer. J Contemp Brachytherapy 2018; 10: 17-25.

15. Rao BS, Das P, Subramanian BV et al. A comparative analysis of two different dose fractionation regimens of high dose rate intracavitary brachytherapy in treatment of carcinoma of uterine cervix: a prospective randomized study. J Clin Diagn Res 2017; 11: XC06-XC10.

16. Bandyopadhyay A, Basu P, Roy K et al. Treatment of locally advanced carcinoma cervix with special emphasis on brachytherapy: A practice pattern survey among young radiation oncologist of India. South Asian J Cancer 2018; 7: 231-235.

17. Sharma DN, Rath GK, Thulkar $S$ et al. High-dose rate interstitial brachytherapy using two weekly sessions of 10 Gy each for patients with locally advanced cervical carcinoma. Brachytherapy 2011; 10: 242-248.

18. Patel F, Sharma S, Negi P et al. Low dose rate vs. high dose rate brachytherapy in the treatment of carcinoma of the uterine cervix: A clinical trial. Int J Radiat Oncol Biol Phys 1994; 28: 335-341.

19. Orton C, Seyedsadr M, Somnay A. Comparison of high and low dose rate remote afterloading for cervix cancer and the importance of fractionation. Int J Radiat Oncol Biol Phys 1991; 21: $1425-1434$.

20. A randomised controlled trial between two different HDR brachytherapy schedule in locally advanced carcinoma of uterine cervix - full text view. ClinicalTrials.gov [Internet]. Clinicaltrials.gov. 2019 [cited 15 May 2019]. Available from: https://clinicaltrials.gov/ct2/show/NCT02765919

21. Fyles A, Keane TJ, Barton $M$ et al. The effect of treatment duration in the local control of cervix cancer. Radiother Oncol 1992; 25: 273-279.

22. Lanciano RM, Pajak TF, Martz K et al. The influence of treatment time on outcome for squamous cell cancer of the uterine cervix treated with radiation: A patterns-of-care study. Int J Radiat Oncol Biol Phys 1993; 25: 391-397.

23. Khor TH, Tuan JK, Hee SW et al. Radical radiotherapy with high-dose-rate brachytherapy for uterine cervix cancer longterm results. Australas Radiol 2007; 51: 570-577. 